\title{
SPIRALLIKE NONANALYTIC FUNCTIONS
}

\author{
HASSOON AL-AMIRI ${ }^{1}$ AND PETRU T. MOCANU
}

\begin{abstract}
Let $f(z)=u(x, y)+i v(x, y)$ be a complex function defined in the unit disc $U . f$ is said to belong to the class $C^{1}(U)$ if the functions $u(x, y)$ and $v(x, y)$ have continuous first order partial derivatives in $U$. We determine sufficient conditions for functions in the class $C^{1}(U)$ to be univalent and to map $U$ onto spirallike domains. These conditions are similar to those in the analytic case as given by Spaček and Rakhmanov.
\end{abstract}

In a recent paper [1] the second author obtained a sufficient condition for a function of the class $C^{1}$ to be univalent and starlike in the unit disc $U$. In this note we generalize this result by obtaining a sufficient condition for a function $f$ in the class $C^{1}(U)$ to be univalent and to map $U$ onto a spirallike domain.

1. Preliminaries. Let $f$ be a complex function defined in the unit disc $U=\{z$ : $|z|<1\}$. For $z=x+i y \in U$, we put

$$
f(z)=u(x, y)+i v(x, y) .
$$

We say that the function $f$ belongs to the class $C^{1}(U)$ if the real functions $u=\operatorname{Re} f$ and $v=\operatorname{Im} f$ of the real variables $x$ and $y$ have continuous first order partial derivatives in $U$. For $f \in C^{1}(U)$, we denote

$$
D f=z \frac{\partial f}{\partial z}-\bar{z} \frac{\partial f}{\partial \bar{z}} \quad \text { and } \quad \mathscr{D} f=z \frac{\partial f}{\partial z}+\bar{z} \frac{\partial f}{\partial \bar{z}},
$$

where

$$
\frac{\partial}{\partial z}=\frac{1}{2}\left(\frac{\partial}{\partial x}-i \frac{\partial}{\partial y}\right), \quad \frac{\partial}{\partial \bar{z}}=\frac{1}{2}\left(\frac{\partial}{\partial x}+i \frac{\partial}{\partial y}\right)
$$

It is easy to verify the following useful formulas

$$
\begin{aligned}
& \frac{\partial|f|}{\partial \theta}=-|f| \operatorname{Im} \frac{D f}{f}, \quad \frac{\partial|f|}{\partial r}=\frac{|f|}{r} \operatorname{Re} \frac{\mathscr{D} f}{f}, \\
& \frac{\partial}{\partial \theta} \arg f=\operatorname{Re} \frac{D f}{f}, \quad \frac{\partial}{\partial r} \arg f=\frac{1}{r} \operatorname{Im} \frac{\mathscr{D f}}{f} .
\end{aligned}
$$

The Jacobian of $f$ is given by

$$
J f=\left|\frac{\partial f}{\partial z}\right|^{2}-\left|\frac{\partial f}{\partial \bar{z}}\right|^{2} .
$$

If $J f>0$ for $z \in U$, then $f$ is locally a homeomorphism preserving the orientation.

Received by the editors March 5, 1980; presented to the Society, January 5, 1980.

1980 Mathematics Subject Classification. Primary 30C99, 30A10; Secondary 30C45.

Key words and phrases. Univalent, starlike, spirallike, Jacobian, nonanalytic functions.

${ }^{1}$ The first author acknowledges support received from the National Academy of Sciences through its exchange program with the Academy of the Socialist Republic of Romania. 
2. Main result. We recall that a closed curve $C$ is logarithmically spirallike of type $\gamma,|\gamma|<\pi / 2$, if each logarithmic spiral $w(t)$ of the equation

$$
\operatorname{Im}\left[e^{i \gamma} \log w(t)\right]=\text { const, } \quad t \in(-\infty, \infty),
$$

intersects $C$ at a single point. In such case $C$ is a Jordan curve and the domain $D$ bounded by $C$ is a spirallike domain of type $\gamma$, i.e., for each $w_{0} \in D$ the arc of the logarithmic spiral joining $w_{0}$ to the origin lies in $D$.

The following theorem provides a sufficient condition of homeomorphism expressed by the geometrical property of spirallikeness of the level curves of the range of $f$. The technique used in the proof of this theorem is similar to the one employed by Rakhmanov in [2] and [3].

Theorem 1. Let $f \in C^{1}(U)$ and let $\gamma$ be a given real number, $-(\pi / 2)<\gamma<\pi / 2$. If $f$ satisfies the following conditions,

(i) $f(0)=0$ and $f(z) \neq 0$, for all $z \in U \backslash\{0\}$,

(ii) $J f(z)>0$, for all $z \in U$,

(iii) $\operatorname{Re}\left[e^{i \gamma} D f(z) / f(z)\right]>0$, for all $z \in U \backslash\{0\}$,

then $f$ is univalent in $U$ and the range $f(U)$ is a logarithmically spirallike domain of type $\gamma$.

Proof. For $r \in(0,1)$ we denote $U_{r}=\{z:|z|<r\}$ and $C_{r}=f\left(\partial U_{r}\right)$. In order to prove that the function $f$ is univalent in $U$, it is sufficient to show that the curves $C_{r}$ are nonintersecting Jordan curves.

Considering the family of spirals $\left(a_{\phi}\right), \phi \in[0,2 \pi)$, where $a_{\phi}$ has the parametric equation

$$
a_{\phi}: w=w_{\phi}(t), \quad t \in(-\infty, \infty)
$$

and

$$
w_{\phi}(t)=e^{t \cos \gamma} e^{i(\phi-t \sin \gamma)} .
$$

It is clear that through each point $w \in C \backslash\{0\}$ passes only one spiral of the family $\left(a_{\phi}\right)$. Hence, for $z=\mathrm{re}^{i \theta}, 0<r<1,0<\theta<2 \pi$, the equation

$$
f(z)=w_{\phi}(t)
$$

determines a unique $\phi=\phi(r, \theta) \in[0,2 \pi)$.

Let us first prove that $C_{r}$ is a Jordan curve for each $r \in(0,1)$. This can be achieved by showing that

$$
\frac{\partial \phi}{\partial \theta}>0, \quad \text { for } \theta \in[0,2 \pi)
$$

and

$$
\underset{0<\theta<2 \pi}{\operatorname{Var}} \phi(r, \theta)=2 \pi
$$

From (3) and (4) we get

$$
\begin{gathered}
t \cos \gamma=\log |f(z)|, \\
\phi-t \sin \gamma=\arg f(z)
\end{gathered}
$$


Consequently

$$
\phi=\arg f(z)+\operatorname{tg} \gamma \cdot \log |f(z)| .
$$

By using (1) and (2), we obtain from (9)

$$
\frac{\partial \phi}{\partial \theta}=\operatorname{Re} \frac{D f(z)}{f(z)}-\operatorname{tg} \gamma \cdot \operatorname{Im} \frac{D f(z)}{f(z)}=\frac{1}{\cos \gamma} \operatorname{Re}\left[e^{i \gamma} \frac{D f(z)}{f(z)}\right] .
$$

Hence by condition (iii) and the above we get (5). Furthermore, condition (i) implies that the curves $C_{r}, r \in(0,1)$, are homotopic in the domain $\mathbf{C} \backslash\{0\}$. Thus they have the same index with respect to the origin, i.e., ind ${ }_{0} C_{r}=$ constant for all $r \in(0,1)$. By condition (ii) the function $f$ is univalent and preserves the orientation in a neighborhood of the origin. This implies the existence of $r_{0} \in(0,1)$ such that ind $_{0} C_{r}=1$ for $r<r_{0}$. Hence the total variation of the argument along $C_{r}$ is $2 \pi$; that is,

$$
\underset{0<\theta<2 \pi}{\operatorname{Var}} \arg f\left(r e^{i \theta}\right)=2 \pi, \quad r \in(0,1) .
$$

Now (9) and (10) yield

$$
\underset{0<\theta<2 \pi}{\operatorname{Var}} \phi(r, \theta)=\underset{0<\theta<2 \pi}{\operatorname{Var}} \arg f\left(r e^{i \theta}\right)=2 \pi
$$

which is (6). Thus for each $r \in(0,1), C_{r}$ is a simple spirallike curve of type $\gamma$.

To complete the proof of the theorem we need only show that $C_{r} \cap C_{r^{\prime}}=\varnothing$ whenever $r \neq r^{\prime}, r, r^{\prime} \in(0,1)$. Fix a value $\phi \in[0,2 \pi)$. The system

$$
f(z)=w_{\phi}(t), \quad|z|=r, 0<r<1,
$$

yields a unique $z=\mathrm{re}^{i \theta}, \theta=\theta(r)$, and a unique $t=t(r, \theta)=t(r)$. It follows that our assertion is equivalent to showing

$$
\frac{d t}{d r}>0, \text { for } r \in(0,1) \text {. }
$$

By differentiating (7) and (8) with respect to $r$ and using (1) and (2) we obtain

$$
\begin{aligned}
\frac{d t}{d r} \cos \gamma & =\frac{1}{r} \operatorname{Re} \frac{\mathscr{D} f(z)}{f(z)}-\frac{d \theta}{d r} \operatorname{Im} \frac{D f(z)}{f(z)}, \\
-\frac{d t}{d r} \sin \gamma & =\frac{1}{r} \operatorname{Im} \frac{\mathscr{D} f(z)}{f(z)}+\frac{d \theta}{d r} \operatorname{Re} \frac{D f(z)}{f(z)} .
\end{aligned}
$$

Eliminating $d \theta / d r$ from the above system of equations we get

$$
\begin{aligned}
\left(\cos \gamma \operatorname{Re} \frac{D f(z)}{f(z)}-\right. & \left.\sin \gamma \operatorname{Im} \frac{D f(z)}{f(z)}\right) \frac{d t}{d r} \\
& =\frac{1}{r}\left(\operatorname{Re} \frac{D f(z)}{f(z)} \operatorname{Re} \frac{\mathscr{D} f(z)}{f(z)}+\operatorname{Im} \frac{D f(z)}{f(z)} \operatorname{Im} \frac{\mathscr{D} f(z)}{f(z)}\right) .
\end{aligned}
$$

Hence

$$
\frac{d t}{d r} \operatorname{Re}\left[e^{i \gamma} \frac{D f(z)}{f(z)}\right]=\frac{1}{r} \operatorname{Re} \frac{D f(z) \cdot \overline{\Phi f(z)}}{f(z) \cdot \overline{f(z)}}
$$


However, it is easy to verify that $\operatorname{Re}(D f(z) \cdot \overline{\mathscr{D} f(z)})=r^{2} J f(z)$. Consequently we have the formula

$$
|f(z)|^{2} \frac{d t}{d r} \operatorname{Re}\left[e^{i \gamma} \frac{D f(z)}{f(z)}\right]=r J f(z) .
$$

From (ii), (iii) and (12) we realize (11). This shows that the curves $C_{r}$ do not intersect each other. Hence $f$ is univalent in $U$ and $f(U)$ is spirallike for each $r \in(0,1)$. Moreover, $f\left(U_{r}\right) \subset f\left(U_{r}\right)$ for $0<r<r^{\prime}<1$. It follows that the domain $f(U)$ is spirallike of type $\gamma$. This completes the proof of Theorem 1 .

3. Remarks. (a) For $\gamma=0$, Theorem 1 reduces to the starlike case [1].

(b) If $f(z)$ is analytic in $U$, then $D f(z)=\mathscr{D} f(z)=z f^{\prime}(z)$.

Thus Theorem 1 and its special case as indicated in part (a) above become the familiar spirallike case of Spaček [4] and the starlike case, respectively.

4. Example. In this section we discuss an example of a function in $C^{1}(U)$ satisfying the conditions of Theorem 1 .

Consider

$$
f(z)=\frac{z}{\left[(1-z)^{\alpha}(1-\bar{z})^{\beta}\right]^{\cos \gamma e^{-\gamma}}},
$$

$|\gamma|<\pi / 2,0 \leqslant \alpha \leqslant 2$ and $\beta$ is a real number such that

$$
|\beta| \leqslant \beta(\alpha)=\frac{1}{\cos \gamma}\left[1-\sqrt{1-\alpha(2-\alpha) \cos ^{2} \gamma}\right] .
$$

Such functions satisfy the conditions of Theorem 1 .

$$
\frac{D f(z)}{f(z)}=1+\frac{\alpha z \cos \gamma e^{-i \gamma}}{1-z}-\frac{\beta \bar{z} \cos \gamma e^{-i \gamma}}{1-\bar{z}},
$$

hence

$$
\operatorname{Re}\left[e^{i \gamma} \frac{D f(z)}{f(z)}\right]>\cos \gamma\left[1-\frac{\alpha-\beta}{2}\right] \geqslant 0
$$

for all $\alpha$ and $\beta$ satisfying the condition

$$
0 \leqslant \alpha-\beta \leqslant 2 .
$$

Let $A=\alpha \cos \gamma e^{-i \gamma}, B=\beta \cos \gamma e^{-i \gamma}$. Then $|\partial f / \partial z|>|\partial f / \partial \bar{z}|$ if and only if $|1+(A-1) z|>|B z|$ for all $z \in U$. However $|A-1|<1$ and consequently $J f(z)$ $>0$ provided

$$
|1+(A-1) z|>1-|A-1| \geqslant|B|>|B z|
$$

that is

$$
|A-1|+|B|<1
$$

Direct calculations can show that (15) and (13) are equivalent. Thus the condition $J f(z)>0$ for all $z \in U$ is satisfied.

Obviously condition (iii) of Theorem 1 will be satisfied if one can show that every $(\alpha, \beta)$ satisfying (13) must also satisfy (14). It is clear that the region 
containing $\{(\alpha, \beta)\}$ satisfying (13) is bounded by two branches of the hyperbolas

$$
\beta(\alpha)= \pm \frac{1}{\cos \gamma}\left[1-\sqrt{1-\alpha(2-\alpha) \cos ^{2} \gamma}\right]
$$

These hyperbolas have the same axis of symmetry, $\alpha=1$, and they intersect at $(0,0)$ and $(2,2)$. Since $\beta^{\prime}(0)= \pm \cos \gamma$, the assertion follows at once. This completes the discussion of our example.

5. More spirallike functions. In this section we state two results on the univalency of the Archimedean and the hyperbolic spirallike functions. Before we state these results we need to recall the definition of these terms.

Let $\left(\sigma_{\phi}\right)$ be the parametric family of spiral arcs defined by

$$
\sigma_{\phi}: w=w_{\phi}(t)=t e^{i(t+\phi)}, \quad t \in(0, \infty), \phi \in[0,2 \pi) .
$$

It is clear that through each point $w \in C \backslash\{0\}$ passes only one spiral of the family $\left(\sigma_{\phi}\right)$.

We say that $D$ is an Archimedean spirallike domain if for each $w \in D, w \neq 0$, the part of the spiral arc $\sigma_{\phi}$ joining the origin to the point $w$ lies entirely in $D$. Similarly if the family $\left(\sigma_{\phi}\right)$ is defined by $\sigma_{\phi}: w=w_{\phi}(t)=\mathrm{e}^{i(t+\phi)} / t, t \in(0, \infty), \phi \in[0, w]$, we say $D$ is a hyperbolic spirallike domain if for each point $w \in D, w \neq 0$, the point of the spiral arc $\sigma_{\phi}$ joins the part $w$ to the origin lies entirely in $D$.

THEOREM 2. If $f \in C^{1}(U)$ verifies the conditions

(i) $f(0)=0, f(z) \neq 0, z \in U \backslash\{0\}$,

(ii) $J f(z)>0, z \in U$,

(iii) $\operatorname{Re}[(1-i|f(z)|) D f(z) / f(z)]>0$

then $f$ is univalent in $U$ and $f(U)$ is an Archimedean spirallike domain.

TheOREM 3. If $f \in C^{1}(U)$ verifies the conditions

(i) $f(0)=0, f(z) \neq 0, z \in U \backslash\{0\}$,

(ii) $J f(z)>0, z \in U$,

(iii) $\operatorname{Re}[(|f(z)|+i) D f(z) / f(z)]>0, z \in U \backslash\{0\}$,

then $f$ is univalent and $f(U)$ is a hyperbolic spirallike domain.

When $f(z)$ is analytic in $U$, Theorems 2 and 3 are due to Rakhmanov [2] and [3].

\section{REFERENCES}

1. Petru T. Mocanu, Starlikeness and convexity for nonanalytic functions in the unit disc, Mathematica (Cluj) (to appear).

2. B. N. Rakhmanov, On the theory of schlicht functions, Dokl. Akad. Nauk SSSR 91 (1953), 729-732. (Russian)

3.

4. L. Spaček, Contribution à la théorie des fonctions univalentes, Casopis Pèst. Mat. 62 (1933), 12-19.

Department of Mathematics, Bowling Green State University, Bowling Green, Ohio 43403

Department of Mathematics, The Babis Bolya University, Cluj, Romania 